DOI:

$10.1038 /$ nphys 860

\title{
An attractive theory
}

Since the earliest observation that a lump of lodestone attracts iron, around 600 BC, magnetism has attracted numerous philosophers and physicists eager to understand its secrets. Yet the physical explanation came only with the development of quantum mechanics in the 1920 s - after all, magnetism is a purely quantum effect arising from the 'spin' property of the electron. By convention, we say that the spin points either up or down. This means that there are two distinct spin states instead of one; hence, certain theories were out by a factor of two with respect to experiments.

Following a series of papers published in the years 1925 to 1927 - during which quantum mechanics was developed, interpreted and applied to atoms with more than one electron outside a closed shell - Werner Heisenberg solved the mystery of ferromagnetism using the concept of spin plus the exclusion principle formulated by Wolfgang Pauli, which states that two electrons with the same energy and momentum cannot occupy the same quantum state. In other words, two electrons with the same energy but different spins can lie in the same orbital. The facts that an electron has spin, as well as charge, and that two identical electrons must occupy different states, are the keys to the periodic table.

Until then, the force aligning the electron spins could not be explained in terms of known interactions, none of which was strong enough. In the words of Paul Dirac: "the solution of this difficulty [...] is provided by the exchange (austausch) interaction of the electrons, which arises owing to the electrons being indistinguishable one from another. Two electrons may change places without our knowing it, and the proper allowance for the possibility of quantum jumps of this nature, which can be made in a treatment of the problem by quantum mechanics, gives rise to the new kind of interaction. The energies involved, the so-called exchange energies, are quite large."

By applying such an energy tax on indistinguishable particles, Heisenberg proposed a model that counted up all the spins and included the exchange interaction between nearest neighbours only; for example, in a linear chain of spins, only two neighbouring spins would count, in a square lattice, four. When these were summed, Heisenberg found a ground state (lowest-energy configuration) in which all the spins of the electrons lined up in parallel - that is, a ferromagnetic state without the need for any external magnetic field.

May Chiao, Senior Editor, Nature Physics 